\title{
Towards Improving the Prospects for Coordinated Gravitational-Wave and Electromagnetic Observations
}

\author{
Ilya Mandel ${ }^{1}$, Luke Z. Kelley ${ }^{2}$ and Enrico Ramirez-Ruiz ${ }^{3}$ \\ ${ }^{1}$ School of Physics \& Astronomy, University of Birmingham, Edgbaston, B15 2TT, UK \\ email: imandelestar.sr.bham.ac.uk \\ ${ }^{2}$ Harvard-Smithsonian Center for Astrophysics, Cambridge, MA 02138, USA \\ ${ }^{3}$ Dept. of Astronomy \& Astrophysics, University of California, Santa Cruz, CA 95064, USA
}

\begin{abstract}
We discuss two approaches to searches for gravitational-wave (GW) and electromagnetic (EM) counterparts of binary neutron-star mergers. The first approach relies on triggering archival searches of GW detector data based on detections of EM transients. Quantitative estimates of the improvement to GW detector reach due to the increased confidence in the presence and parameters of a signal from a binary merger gained from the EM transient suggest utilizing other transients in addition to short gamma-ray bursts. The second approach involves following up GW candidates with targeted EM observations. We argue for the use of slower but optimal parameter-estimation techniques and for a more sophisticated use of astrophysical prior information, including galaxy catalogues to find preferred follow-up locations.
\end{abstract}

Advanced ground-based gravitational-wave detectors LIGO (Harry \& the LIGO science collaboration 2010) and Virgo (Virgo Collaboration 2009) are expected to begin taking data around 2015, operating at a sensitivity approximately a factor of 10 better than their initial counterparts. Gravitational waves (GWs) emitted during the late inspirals and mergers of compact-object binaries will be one of the main sources for these detectors. Although predictions for merger rates of binary neutron stars (BNSs) and neutron-starblack-hole (NSBH) binaries are highly uncertain (Mandel \& O'Shaughnessy 2010), we may expect detections at a rate between one per few years and a few hundred per year, with perhaps a few tens of detections per year being most likely (Abadie et al. 2010a).

These detections would usher in an era of genuine gravitational-wave astronomy, with GWs being used as another tool to observe the sky. There has been a lot of discussion in the literature of the promise of multi-messenger observations (e.g., Bloom 2009). In fact, the recent focus on multi-messenger astronomy has been so exclusive that it is worthwhile to recall that a significant amount of astrophysics can be extracted from GW observations alone, since the GW signal encodes the masses and spins of the binary components, and can be used to probe astrophysics, strong-field gravity, and cosmology even in the absence of electromagnetic (EM) observations of counterparts to GW events. Nonetheless, there is no doubt that observing both EM and GW counterparts of the same event would be of great astrophysical significance, and would allow us to settle crucial questions such as the origins of short GRBs. Here we describe some thoughts about (1) future possibilities for triggered searches of archival GW data based on EM transients observed during surveys, and (2) possible improvements to recently started efforts to follow up GW triggers with targeted EM observations.

GW searches triggered on EM transients. The LIGO Scientific Collaboration and the Virgo Collaboration have previously used short, hard Gamma-Ray Bursts (SGRBs) 
as triggers to search for compact binary coalescences in GW detector data (Abadie et al. 2010b). SGRBs are believed to be associated with relativistic jets from BNS or NS-BH mergers (Lee \& Ramirez-Ruiz 2007). However, the impact of the SGRB observation on gaining confidence in the presence of a GW signature from a binary merger has not been quantitatively estimated. In fact, most of the observed SGRBs are too distant for us to detect the associated GW signal; the average luminosity distance for the 16 SGRBs with confident host identifications and redshift measurements compiled by Berger (2010) is approximately $5 \mathrm{Gpc}$, which exceeds the anticipated event horizon of Advanced LIGO by more than a factor of 10. Hence, even when an SGRB is detected, it is unlikely that a gravitational-wave counterpart is observable, so the detectability threshold is not lowered by as much as could be anticipated.

We have developed a Bayesian framework to incorporate accurately the information from an EM transient in order to quantify the benefits of triggered searches for improving the detector reach. The detection of an EM transient which may originate in a compact-object binary merger will increase the a priori probability that a given stretch of data from the LIGO-Virgo ground-based gravitational-wave detector network contains a signal from a binary coalescence. That increase depends on the confidence that the transient is associated with a binary coalescence, the probability that the coalescence occurs within the detection volume, and the accuracy of the merger-time reconstruction from the transient. Additional information contained in the EM signal, such as the sky location, inclination or distance to the source, can further rule out false alarms and thus lower the necessary threshold for a detection. For a fixed false-alarm probability, the EM transient can reduce the signal-to-noise ratio threshold for detection by up to $\sim 60-80 \%$ for optimal searches, depending on the assumptions made. If the untriggered search is incoherent while the triggered search is optimized because of the known sky location (Harry \& Fairhurst 2011), the improvement can be even more significant.

Preliminary results suggest that optical signatures from r-processes in the tidal tails (also known as "kilonovæ") could be one of the most promising triggers. They are likely to be sufficiently numerous in all-sky surveys like the LSST, differentiable from other transients based on the light-curve profiles if the observational cadence is sufficiently high, and could yield moderate improvements in the detectability thresholds.

EM followups of GW candidate events. The benefits of following up GW candidates with targeted EM observations have long been recognized (Finn et al. 1999). Recently the first pilot programme for following up GW candidate events has been activated (Abadie et al. 2011). There are two significant complications in this effort: (a) the prompt analysis of GW candidates, including detection confidence and sky location; and (b) the large uncertainty in the positional reconstruction of GW detections, which could encompass tens or even a few hundred square degrees on the sky depending on the candidate and the detector network configuration (Van Der Sluys et al. 2008b).

A significant amount of effort has been expended to allow for very rapid processing of GW data with the aim of achieving latencies of only a few seconds or tens of seconds to identify GW candidates (Cannon et al. 2011). Timing triangulation between different GW interferometers comprising the network has so far been used to localize rapidly the source on the sky (Fairhurst 2009). Such incoherent use of detector data permits rapid analysis, but is likely to yield sub-optimal results. The "need for speed" can be over-stated: it is simply not necessary for many of the possible EM counterparts. For example, off-axis optical afterglows outside the jet opening angle will only peak on time scales greater than $\sim 1$ day (van Eerten \& MacFayden 2011). We may hope that if we are inside the jet opening angle the GRB will be picked up by a mission like SwIFT; but 
even if not, the optical signal has decay time-scales of at least several hours. Time-scales for lower-frequency follow-ups such as radio are even longer. We thus have the luxury of applying slower parameter-estimation techniques that perform a coherent analysis of all of the detector data (Van Der Sluys et al. 2008b), and should be able to improve sky localization and quantify better the confidence regions.

Even with slower, optimal data-analysis techniques, the confidence regions are much larger than the fields of view of typical follow-up instruments. Efforts to reduce the GW sky error box have relied on a galaxy catalogue (Nuttall \& Sutton 2010). In particular, pixels within the error box have been re-weighted by the blue-light luminosity of the galaxies contained in them (Abadie et al. 2011). While that approach is a reasonable first cut, it could be problematic for three reasons. First, blue-light luminosity is a proxy for the star-formation rate, but mergers could be significantly delayed relative to starformation episodes, and red elliptical galaxies may substantially contribute to present-day merger rates (O'Shaughnessey et al. 2010). Secondly, galaxy catalogues are unlikely to be complete to $\sim 400 \mathrm{Mpc}$ (the horizon distance of Advanced LIGO for optimally located and oriented coalescing neutron-star binaries). Finally, some fraction of mergers may happen outside host galaxies altogether, if the progenitor binary experienced large supernovæ kicks (Kelley et al. 2010). An ongoing study by Vousden et al. (2012) aims to account for these shortcomings by employing more sophisticated astrophysical priors.

Despite the improvements mentioned above, finding an electromagnetic counterpart of a GW candidate will remain extremely challenging, as discussed by Metzger \& Berger (2011). Coordinated observing among several facilities may be required to cover the large uncertainty region with smaller field-of-view instruments. Another alternative that may be worth investigating is the deployment of a network of inexpensive robotic telescopes specifically with the goal of following up GW candidates, though it will be difficult to detect any but the closest afterglows. While it is impossible to guarantee that an EM counterpart to given candidate would be found, we can still strive to maximize the probability of a successful follow-up with a view to ensuring that a sufficient fraction of triggers are followed up to make at least some multi-messenger observations.

\section{References}

Abadie, J., et al. 2010a, Classical and Quantum Gravity, 27, 173001

Abadie, J., et al. 2010b, ApJ, 715, 1453

Abadie, J., et al. 2011, ArXiv e-prints, 1109.3498

Berger, E. 2010, ApJ, 722, 1946

Bloom, J. S., et al. 2009, ArXiv e-prints, 0902.1527

Cannon, K., et al. 2011, ArXiv e-prints, 1107.2665

Fairhurst, S. 2009, New Journal of Physics, 11, 123006

Finn, L. S., Mohanty, S. D., \& Romano, J. D. 1999, Phys. Rev. D, 60, 121101

Harry, G. M., the LIGO Scientific Collaboration. 2010, Class. Quant. Grav., 27, 084006

Harry, I. W. \& Fairhurst, S. 2011, Phys. Rev. D, 83, 084002

Kelley, L. Z., et al. 2010, ApJ (Letters), 725, L91

Lee, W. H. \& Ramirez-Ruiz, E. 2007, New Journal of Physics, 9, 17

Mandel, I. \& O'Shaughnessy, R. 2010, Class. Quant. Grav., 27, 114007

Metzger, B. D. \& Berger, E. 2011, ArXiv e-prints, 1108.6056

Nuttall, L. K. \& Sutton, P. J. 2010, Phys. Rev. D, 82, 102002

O'Shaughnessy, R., Kalogera, V., \& Belczynski, K. 2010, ApJ, 716, 615

van der Sluys, M. V., et al. 2008, ApJ, 688, L61

van Eerten, H. J. \& MacFadyen, A. I. 2011, ApJ 733, L37

Virgo Collaboration. 2009, Technical Report VIR-0027A-09 